\title{
Enhanced voltammetric determination of dopamine using a glassy carbon electrode modified with ionic liquid-functionalized graphene and carbon dots
}

\author{
Xuming Zhuang ${ }^{1} \cdot$ Haihua Wang ${ }^{1}$ - Tao He ${ }^{1} \cdot$ Lingxin Chen $^{1,2}$
}

Received: 21 May 2016 / Accepted: 4 October 2016/Published online: 11 October 2016

(C) Springer-Verlag Wien 2016

\begin{abstract}
The authors describe a dopamine (DA) sensor based on a glassy carbon electrode modified with a composite film composed of carbon dots (C-dots) and graphene functionalized with an ionic liquid. The C-dots were functionalized with carboxy groups whose negative charge promotes electrostatic attraction to the protonated amino groups in DA. The presence of an imidazole cation in the IL facilitates interaction with the $\mathrm{C}$-dots and DA via electrostatic interactions and $\pi$ stacking forces. Under optimal conditions, the modified GCE display improved electrochemical response to DA compared to a bare GCE, or a GCE modified with C-dots or IL-graphene only. The oxidation current, measured best at a potential of $0.22 \mathrm{~V}$ (vs. $\mathrm{Ag} / \mathrm{AgCl}$ ) is linearly related to the DA concentration in the 0.1 to $600 \mu \mathrm{M}$ range, with a $30 \mathrm{nM}$ detection limit at a signal-to-noise ratio of 3 . Ascorbic acid does not interfere even in large excess, and the sensor is stable for at least a month. The modified GCE was applied to the determination of DA in spiked fetal bovine serum and gave satisfactory results.
\end{abstract}

Keywords Dopamine $\cdot$ Ionic liquid $\cdot$ Graphene $\cdot$ Carbon dot

Electronic supplementary material The online version of this article (doi:10.1007/s00604-016-1971-8) contains supplementary material, which is available to authorized users.

Lingxin Chen

lxchen@yic.ac.cn

1 College of Chemistry and Chemical Engineering, Yantai University, Yantai 264005, China

2 Key Laboratory of Coastal Environmental Processes and Ecological Remediation, Yantai Institute of Coastal Zone Research, Chinese Academy of Sciences, Yantai 264003, China

\section{Introduction}

Dopamine (DA) behaves as a vital neurotransmitter in the human brain playing a significant role in the renal, cardiovascular, central nervous and hormonal systems $[1,2]$. When an occurrence of an anomaly pertaining to the dopamine concentration presents itself, it is most likely linked to several neurological disorders such as parkinsonism, epilepsy and schizophrenia [3]. In addition, DA is available as an intravenous medication that acts on the sympathetic nervous system. It can produce noticeable effects by increasing heart rate and blood pressure [4]. Therefore, detection and quantification of DA in vivo/vitro is a subject of great importance in medical practice. Various methods, such as liquid chromatography [5], spectroscopy [6], fluorescence [7] and electrochemical analysis $[8,9]$ have been implemented for the assay of DA. Among them, electrochemical analysis has received great interest due to its considerably high sensitivity, rapid response, low cost and ease of operation. Many attempts have been made to develop chemically modified electrodes with a variety of materials for the selective determination of DA; the most commonly used materials are carbon nanomaterials and metal oxides due to their internal excellent catalytic properties, such as carbon nanotubes [10], graphene [11], graphene oxide (GO) [12], $\mathrm{MnO}_{2}$ [13], $\mathrm{Cu}_{2} \mathrm{O}$ [14], $\mathrm{TiO}_{2}$ [15], and $\mathrm{Fe}_{3} \mathrm{O}_{4}$ [16].

Being a two-dimensional carbon material, graphene has demonstrated a myriad of beneficial characteristics [17]. For instance, graphene has been shown to display unique physical and chemical properties in bioelectronics and biosensing [18]. Graphene has exceptional thermal, chemical, and mechanical properties [19], as well as high specific surface area and excellent electrical conductivity [20]. These unique properties hold great promise for potential applications in technological fields such as nanoelectronics, sensors, nanocomposites, batteries, supercapacitors and hydrogen storage [21]. One 
important issue concerning its application is molecular aggregation, in which graphene sheets tend to form agglomerates via van der Waals interactions [22]. Aggregation can be reduced by covalent or non-covalent attachment of other molecules or polymers onto the sheets. It is imperative to introduce desirable substances to graphene in order to elucidate novel properties. Due to their wide solubility and introduction of a surface charge, ionic liquid-functionalized graphene (IL-graphene) sheets with good dispersibility and long-term stability in various solvents have been synthesized by the Niu group [23]. In addition, IL-graphene based electrochemical sensors and biosensors have also been extensively reported in literature for direct electron transfer of various redox enzymes and the detection of various types of compounds, such as p53 gene [24], bovine serum albumin [25], bovine hemoglobin [26], and rutin [27].

Traditional semiconductor quantum dots (such as $\mathrm{CdSe}, \mathrm{CdTe}$ ect.) are commonly synthesized with the use of toxic heavy metals, limiting their widespread use in biological applications [28]. These heavy metals are hazardous to the environment and pose long term toxicity concerns. Compared with conventional semiconductor quantum dots or metal quantum dots, carbon dots (Cdots) are a new class of carbon nanomaterials that are superior due to their low cytotoxicity, excellent biocompatibility, facile synthesis, cost-effective, remarkable conductivity, high aqueous solubility, and favorable optical properties. The C-dots can provide an abundant source of carboxyl groups and/or amino groups at the surface that can significantly enhance the redox response of dopamine [29].

Herein, a kind of compound consisting of carbon dots and IL-graphene was fabricated. It can be implemented for detection of DA and it also shows a wide linear range, higher sensitivity, lower detection limit, better reproducibility, and better stability. In addition, the sensor also has a strong anti-interference ability for potential interference substances. Under optimal conditions, differential pulse voltammetry (DPV) peak current and the concentration of DA in the range from 0.1 to $600 \mu \mathrm{M}$ and its detection limit is $30 \mathrm{nM}$.

\section{Experimental section}

\section{Reagents and instrumentations}

GO was purchased from Nanjing XFNano Materials Technology Company (Nanjing, China, http://www.xfnano. com). Ascorbic acid (AA), DA, 1-methylimidazole and 2bromoethylamine hydrobromide were obtained from Sigma (Shanghai, China, http://www.sigmaaldrich.com/chinamainland.html). All reagents were analytical grade reagents. Nanopure deionized and distilled water $\left(18.2 \mathrm{M} \Omega . \mathrm{cm}^{-1}\right)$ was used throughout all experiments.

Electrochemical studies were performed with a $\mathrm{CHI}$ 660D instrument (Chenhua Instruments, Shanghai, China). A modified glassy carbon electrode (GCE) served as the working electrode, an $\mathrm{Ag} / \mathrm{AgCl}$ (saturated $\mathrm{KCl}$ solution) was used as the reference electrode, and a Pt wire was employed as an auxiliary electrode. The UV-visible absorption was registered on a Thermo Scientific NanoDrop 2000/2000C spectrophotometer. Scanning electron microscopy (SEM) was performed on a S4800 electron microscope (Hitachi, Ltd., Japan). Transmission electron microscopy (TEM) was performed on a JEM-2100F electron microscope (JEOL, Ltd., Japan) at $300 \mathrm{kV}$. All experiments were conducted at room temperature.

\section{Synthesis of C-dots/IL-graphene}

IL-graphene was prepared according to Niu's method [23] and the C-dots were prepared according to Francesca Arcudi's method [30]. A typical procedure to synthesis C-dots/ILgraphene is as follows: $1 \mathrm{mg}$ IL-graphene was added to $10 \mathrm{~mL} \mathrm{C}$-dots solution $\left(2.0 \mathrm{mg} \mathrm{mL}^{-1}\right)$. Subsequently, the composite was placed into a Teflon-lined stainless steel autoclave for $4 \mathrm{~h}$ at $90{ }^{\circ} \mathrm{C}$, and then $\mathrm{C}$-dots/IL-graphene was obtained.

\section{Electrode preparation}

First, the GCE was polished to a mirror-like surface using $0.3 \mu \mathrm{m}$ and $0.05 \mu \mathrm{m} \gamma$-alumina slurry. The GCE was successively washed by water, ethanol and distilled water in an ultrasonic bath and dried in air before use. The obtained C-dots/ IL-graphene nanocomposite solution of $5 \mu \mathrm{L}$ was dropped
Scheme 1 Schematic illustration of the enhanced electrochemical detection strategy for DA based on C-dots/IL-graphene composites modified on the GCE

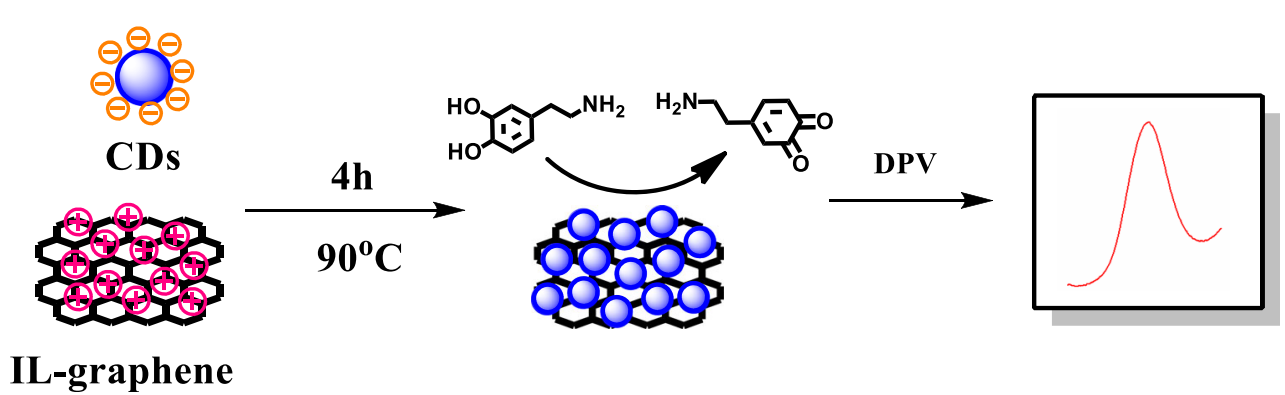


Fig. 1 a SEM image of ILgraphene. b SEM image of Cdots/IL-graphene. c TEM image of C-dots/IL-graphene. d UV-vis absorption and fluorescence emission spectra of C-dots intensity

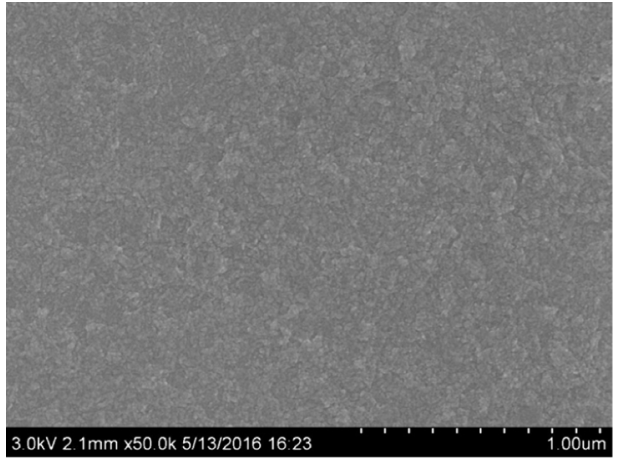

a

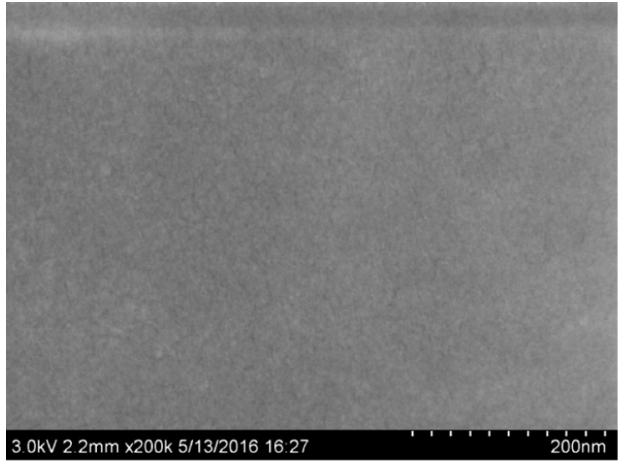

b

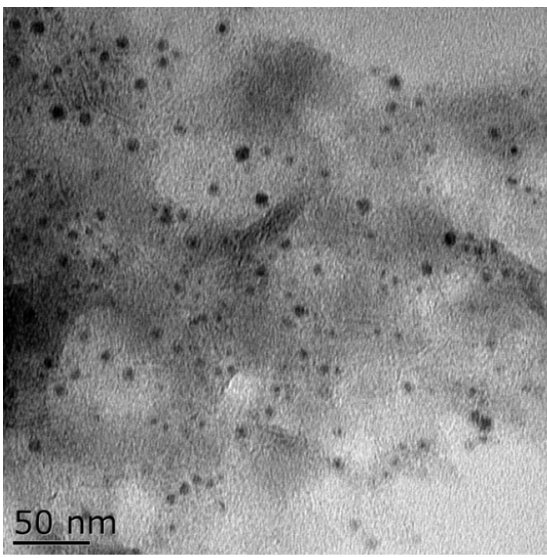

C

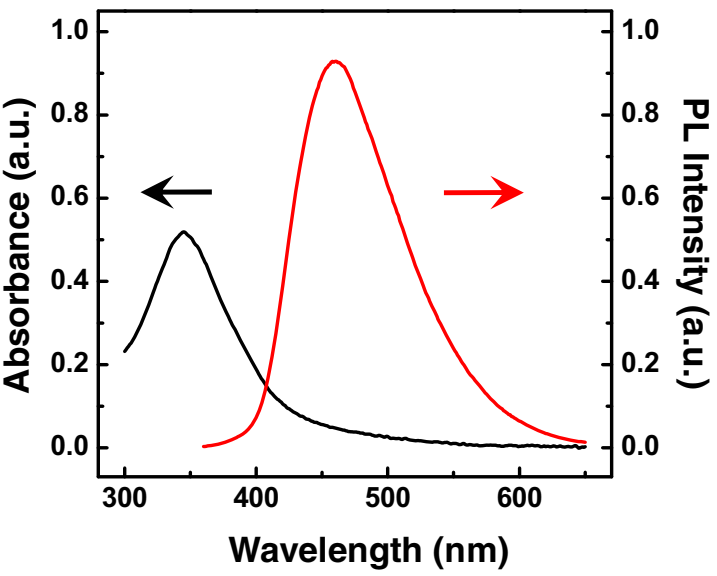

d onto the surface of the GCE and slowly dried in ambient conditions to form C-dots/IL-graphene modified GCE (Cdots/IL-graphene/GCE). Subsequently, it was rinsed with water to physically remove adsorbed species before use. The sensing strategy for DA is illustrated in Scheme 1. Cyclic voltammetry $(\mathrm{CV})$ was performed to characterize the different modified electrodes in the $\mathrm{pH} 6.5$ phosphate buffer containing different concentrations at a scan rate of $0.1 \mathrm{~V} \mathrm{~s}^{-1}$. Finally, the DPV signals were recorded in the range of $-0.2 \mathrm{~V}$ to $+0.6 \mathrm{~V}$.

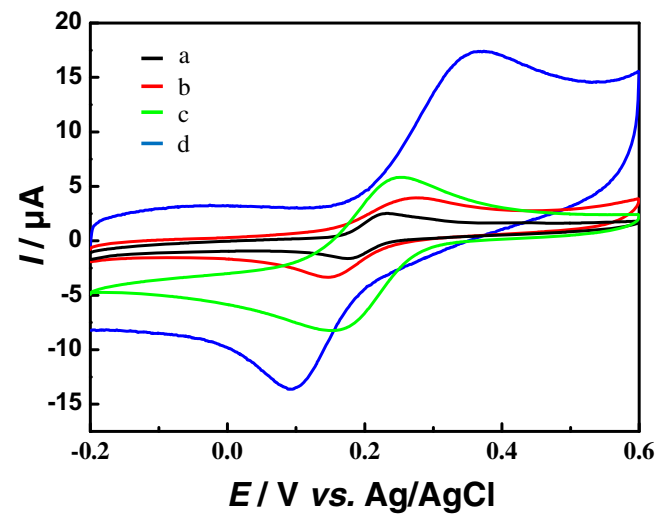

Fig. $2 \mathrm{CV}$ of $0.10 \mathrm{mM}$ DA recorded on bare GCE (a), C-dots/GCE (b), IL-graphene/GCE (c), and C-dots/IL-graphene/GCE (d) in the $\mathrm{pH} 6.5$ phosphate buffer (scan rate: $0.1 \mathrm{~V} \mathrm{~s}^{-1}$ )

\section{Results and discussion}

\section{Characterization of IL-graphene, C-dots, and C-dots/IL-graphene/GCE}

The morphology of IL-graphene (Fig. 1a) and C-dots/ILgraphene (Fig. 1b) were characterized via SEM, the typically crumpled and wrinkled structure of IL-graphene and C-dots/ IL-graphene were observed. As presented in Fig. 1c, the Cdots/IL-graphene composite was characterized via TEM with sizes ranging from 5 to $10 \mathrm{~nm}$ of C-dots Fig. $1 \mathrm{~d}$ depicts the UV-Visible absorption spectra and the fluorescent emission peak of C-dots. C-dots exhibited a well-defined absorption and emission peak at 340 and $460 \mathrm{~nm}$, respectively.

\section{CV behavior of DA on the C-dots/IL-graphene/GCE}

$\mathrm{CV}$ measurements were tested on the different working electrodes of $10 \mathrm{mM}$ DA including a bare GCE, a C-dots/GCE, an IL-graphene/GCE and a C-dots/IL-graphene/GCE in a pH 6.5 phosphate buffer. As depicted in Fig. 2, the cyclic voltammogram measured of bare GCE shows an oxidation peak current at $2.506 \mu \mathrm{A}$ and a reduction peak at $-1.651 \mu \mathrm{A}$. C-dots were immobilized on the same electrode in which the oxidation 


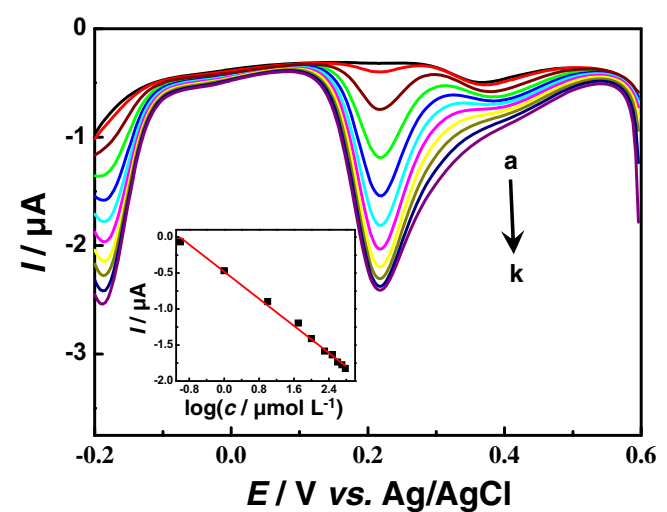

Fig. 3 DPV of DA recorded on C-dots/IL-graphene/GCE with increasing concentration (from a to k: 0, 0.10, 1.0, 10.0, 50.0, 100.0, 200.0, 300.0, $400.0,500.0$, and $600.0 \mu \mathrm{M}$, respectively)

current and the reduction current were observed to have increased by $131.4 \%$ and $400.8 \%$, respectively. On the ILgraphene/GCE, the oxidation current and the reduction current were observed to have increased by $56.6 \%$ and $102.5 \%$, respectively. However, the oxidation and reduction currents are further increased by $591.9 \%$ and $728.0 \%$ at C-dots/ILgraphene/GCE. Many scientists have discussed the possible reaction mechanism of C-dots/IL-graphene/GCE with DA. The C-dots provide a more conductive electrode surface. Cdots have not only increased the stability, but also promoted interactions with amino groups in DA via electrostatic interactions to increase recognition DA with high specificity. [31].

\section{The effect of $\mathrm{pH}$}

The electrochemical redox behavior of DA with C-dots/ILgraphene/GCE in buffer systems of varying $\mathrm{pH}$ values ranging from 3.0 to 8.0 was investigated by $\mathrm{CV}$; results are shown Fig. S1. With an increase of $\mathrm{pH}$ value, results show the oxidation peak potential negatively shifted that indicates protons are being involved in the electrode reaction. A good linear relationship between $\mathrm{E}_{\mathrm{pa}}$ and $\mathrm{pH}$ was constructed with linear regression equation as $\mathrm{E}_{\mathrm{pa}}(\mathrm{V})=-(0.0610 \pm 0.0003) \mathrm{pH}+$
$(0.740 \pm 0.005)\left(n=3, \mathrm{R}^{2}=0.9967\right)$. The slope value of $-61.0 \mathrm{mV} / \mathrm{pH}$ shows that the electron transfer was accompanied by an equal number of protons. At the same time, when the $\mathrm{pH}$ is 6.5 , the oxidation peak current $\left(\mathrm{I}_{\mathrm{pa}}\right)$ of DA was the largest. Therefore, by taking into account the sensitive determination for DA, the phosphate buffer of $\mathrm{pH}=6.5$ was chosen as the optimal $\mathrm{pH}$ acidity in this experiment.

\section{Effects of scan rate}

In order to investigate the reaction kinetics, $\mathrm{CV}$ measurements were performed of $10 \mathrm{mM}$ DA at C-dots/IL-graphene/GCE at varying scan rates, as shown in Fig. S2. As can rate increases, the redox peak current increased correspondingly. Moreover, both the cathodic and anodic peak currents increased with an increase in scan rate from 0.01 to $0.5 \mathrm{~V} \mathrm{~s}^{-1}$. Results indicate that the electro-redox of DA on C-dots/IL-graphene/GCE behaves as a typical diffusion-controlled process [32]. In addition, at higher scan rates, the anode $\left(\mathrm{E}_{\mathrm{pa}}\right)$ and cathode $\left(\mathrm{E}_{\mathrm{pc}}\right)$ peak potential have a linear relationship with the logarithm of scan rate $(\log v)$. The regression equations were: $\mathrm{E}_{\mathrm{pa}}$ $(\mathrm{V})=(0.0744 \pm 0.0002) \log v\left(\mathrm{~V} \mathrm{~s}^{-1}\right)+(0.394 \pm 0.004)$ $\left(n=3, \mathrm{R}^{2}=0.9917\right)$ and $\mathrm{E}_{\mathrm{pc}}(\mathrm{V})=-(0.0351 \pm 0.0003) \log v$ $\left(\mathrm{V} \mathrm{s}^{-1}\right)+(0.0819 \pm 0.0005)\left(\mathrm{n}=3, \mathrm{R}^{2}=0.9910\right)$, respectively. According to the equations by Laviron [33], the relationship between the potential and scan rate can be described and made computations for the electron transfer coefficient $(\alpha=0.58)$ and the number of transfer electron $(n=1.97)$.

\section{Interference effect}

It is well established in literature that the oxidation peak potentials for AA and DA are essentially identical and thus it is difficult to separate these compounds due to their overlapping signals on a bare GCE [31]. This problem can be resolved via electrostatic attractions. The C-dots possess carboxyl groups of a negative charge, preventing anionic AA from reaching the electrode surface. Hence, C-dots/IL-graphene is a anionic
Table 1 Comparing the electrocatalytic performance with other DA electrochemical sensors for the determination of DA

\begin{tabular}{lllll}
\hline Sensors & Method & Linear range $(\mu \mathrm{M})$ & Detection limit $(\mu \mathrm{M})$ & References \\
\hline $\mathrm{Pd}-N C^{\mathrm{a}} / \mathrm{rGO}^{\mathrm{b}}$ & Amperometry & $20-220$ & 7.02 & {$[2]$} \\
$\mathrm{rGO} / \mathrm{Ni}$ & $\mathrm{DPV}$ & $0.05-50$ & 0.01 & {$[3]$} \\
$\mathrm{GNS}-\mathrm{CNTs} / \mathrm{MoS}_{2}$ & $\mathrm{DPV}$ & $0.1-100$ & 0.05 & {$[9]$} \\
{$\left[\mathrm{Ni}(\mathrm{phen})_{2}\right]^{2+} / \mathrm{SWCNTs}^{\mathrm{d}}$} & $\mathrm{DPV}$ & $1-780$ & 1 & {$[10]$} \\
$\mathrm{MoS}_{2} / \mathrm{rGO}$ & DPV & $5-545$ & 0.05 & {$[12]$} \\
$\mathrm{GO}_{\mathrm{MWCNT} / \mathrm{MnO}_{2} / \mathrm{AuNP}^{\mathrm{e}}}$ & Amperometry & $0.5-2500$ & 0.17 & {$[13]$} \\
$\mathrm{pCu}_{2} \mathrm{O} \mathrm{NS}^{\mathrm{f}}-\mathrm{rGO}$ & DPV & $0.05-109$ & 0.015 & {$[14]$} \\
$\mathrm{C}-$ dots/IL-graphene & DPV & $0.1-600$ & 0.03 & This study \\
\hline
\end{tabular}

${ }^{\mathrm{a}}$ palladium nanocubes, ${ }^{\mathrm{b}}$ reduced graphene oxide, ${ }^{\mathrm{c}}$ graphene nanosheets and multiwalled carbon nanotubes, ${ }^{\mathrm{d}}$ the nickel(II)-bis(1,10-phenanthroline) complex and single-walled carbon nanotubes, ${ }^{\mathrm{e}}$ gold nanoparticles, ${ }^{\mathrm{f}}$ porous $\mathrm{Cu}_{2} \mathrm{O}$ nanospheres 
Table 2 Method recoveries for the determination of DA in the fetal bovine serum $(n=5)$

\begin{tabular}{llll}
\hline Spiked $(\mu \mathrm{M})$ & Found $(\mu \mathrm{M})$ & Recovery $(\%)$ & RSD $(\%)$ \\
\hline 0.50 & 0.504 & 100.7 & 1.6 \\
5.0 & 4.98 & 99.5 & 2.0 \\
50 & 50.2 & 100.4 & 1.2 \\
150 & 149.1 & 99.1 & 2.7 \\
\hline
\end{tabular}

exchanger at the GCE surface selectively attracting cationic DA and allowing them to pass through to the electrode surface, while preventing anionic AA from reaching the electrode surface; as a result, AA did not exchange electrons with the electrode. Fig. S3 showed that AA does not interfere.

Furthermore, other influences from common co-existing substances were investigated. When the relative error $\left(\mathrm{E}_{\mathrm{r}}\right)$ exceeded $5 \%$, the tested matter was considered to be an interfering agent. The common interferences in vivo and chemicals similar to DA, such as epinephrine, 3-methoxyphenol, quercetin, vanillin, serotonin, tyramine, and dopa, were tested the disturbance to the defection of DA as shown in Fig. S4. The results revealed these interferences did not response at the Cdots/IL-graphene/GCE. It was found that most ions and common substances at high concentration caused negligible change: $\mathrm{Na}^{+}, \mathrm{K}^{+}, \mathrm{Cl}^{-}, \mathrm{NO}_{3}{ }^{-}, \mathrm{SO}_{4}{ }^{2-}$ (>300 fold); $\mathrm{Ca}^{2+}, \mathrm{Zn}^{2+}$, $\mathrm{Mg}^{2+}$ (150 fold); uric acid, lysine, cysteine and glucose (100 fold). Results indicate that the C-dots/IL-graphene/GCE exhibited enhanced selectivity for DA detection.

\section{Detection of dopamine}

The more sensitive DPV was implemented as the detection method for the C-dots/IL-graphene/GCE device. Under the optimal conditions, the oxidation peak current $\left(\mathrm{I}_{\mathrm{pa}}\right)$ of DA increased with its concentration in the range from $0.1 \mu \mathrm{M}$ to $600.0 \mu \mathrm{M}$. The DPV shown in Fig. 3 was equated by the linear regression equations of $\mathrm{I}_{\mathrm{pa}}(\mu \mathrm{A})=-(0.4706 \pm 0.0001)$ $\operatorname{lgc}(\mu \mathrm{M})-(0.4809 \pm 0.0003)\left(n=3, \mathrm{R}^{2}=0.9939\right)$; the detection limit was calculated to be $30 \mathrm{nM}(\mathrm{S} / N=3)$. It was lower than some previous reports $[34,35]$, illustrating C-dots/ILgraphene/GCE possesses improved sensitivity and a wide linear range. Table 1 showed a comparison of the methods, linear ranges, and detection limits by $\mathrm{Pd}, \mathrm{Ni}$, and other nanomaterials used for electrochemically determining dopamine in a phosphate buffer media.

The repeatability of the sensors was examined by analysis of the same concentration of DA $(0.5 \mathrm{mM})$ using five equally prepared electrodes. The five sensors exhibited similar response and the relative standard deviation (RSD) was $2.8 \%$ (less than $5 \%$ ). When the sensors were stored at $4{ }^{\circ} \mathrm{C}$ after a week, it remained $92.6 \%$ of its initial response. These results indicated the sensors represented excellent repeatability and stability.

\section{Application of the probe}

The utilization of the method in real sample analysis was also investigated by direct analysis of DA in the fetal bovine serum. The ability in DPV determination of DA concentration in the fetal bovine serum based on the repeated DPV responses $(n=5)$ of the diluted analytes was measured. The samples were spiked with a specified concentration of DA and, using the standard addition method, measurements were made of the DA concentration in the pharmaceutical preparations and of the recovery rate of the spiked samples; the results are listed in Table 2.

\section{Conclusions}

A DA sensor based on the C-dots/IL-graphene/GCE electrode with high sensitivity, improved specificity and adequate stability was developed and characterized. Under optimal conditions, selective detection of DA in a linear concentration range of $0.1-600.0 \mu \mathrm{M}$ was obtained at a tolerance limit of $30 \mathrm{nM}$. Furthermore, C-dots/IL-graphene/GCE exhibited enhanced ability to suppress the background current from large excess AA. Additionally, the C-dots/IL-graphene/GCE was applied to the detection of DA with satisfactory results. Therefore, a Cdots/IL-graphene/GCE is a promising analytical platform for the detection of DA.

Acknowledgments This work was financially supported by the National Natural Science Foundation of China (21405132, 21275158) and Young Scholars Fund of Yantai University (Grant HY13B21).

Compliance with ethical standards The author(s) declare that they have no competing interests.

\section{References}

1. Hammami A, Sahli R, Raouafi N (2016) Indirect amperometric sensing of dopamine using a redox-switchable naphthoquinone-terminated self-assembled monolayer on gold electrode. Microchim Acta 183:1137-1144

2. Hsieh YS, Hong BD, Lee CL (2016) Non-enzymatic sensing of dopamine using a glassy carbon electrode modified with a nanocomposite consisting of palladium nanocubes supported on reduced graphene oxide in a nafion matrix. Microchim Acta 183:905-910

3. Kumar MK, Prataap RV, Mohan S, Jha SK (2016) Preparation of electro-reduced graphene oxide supported walnut shape nickel nanostructures, and their application to selective detection of dopamine. Microchim Acta 183:1759-1768

4. Ye N, Neumeyer JL, Baldessarini RJ, Zhen X, Zhang A (2013) Update 1 of: recent progress in development of dopamine receptor subtype-selective agents: potential therapeutics for neurological and psychiatric disorders. Chem Rev 113:PR123-PR178

5. Zhou YP, Yan HL, Xie QJ, Huang SY, Liu JL, Li Z, Ma M, Yao SZ (2013) Simultaneous analysis of dopamine and homovanillic acid 
by high-performance liquid chromatography with wall-jet/thin-layer electrochemical detection. Analyst 138:7246-7253

6. Yusoff N, Pandikumar A, Ramaraj R, Ngee LH, Huang NM (2015) Gold nanoparticle based optical and electrochemical sensing of dopamine. Microchim Acta 182:2091-2114

7. Lin VSY, Lai CY, Huang J, Song SA, Xu S (2001) Molecular recognition inside of multifunctionalized mesoporous silicas: toward selective fluorescence detection of dopamine and glucosamine. J Am Chem Soc 123:11510-11511

8. Liu R, Zeng X, Liu J, Luo J, Zheng Y, Liu X (2016) A glassy carbon electrode modified with an amphiphilic, electroactive and photosensitive polymer and with multi-walled carbon nanotubes for simultaneous determination of dopamine and paracetamol. Microchim Acta 183:1543-1551

9. Mani V, Govindasamy M, Chen SM, Karthik R, Huang ST (2016) Determination of dopamine using a glassy carbon electrode modified with a graphene and carbon nanotube hybrid decorated with molybdenum disulfide flowers. Microchim Acta 183:2267-2275

10. Yan S, Li X, Xiong Y, Wang M, Yang L, Liu X, Zhang C (2016) Simultaneous determination of ascorbic acid, dopamine and uric acid using a glassy carbon electrode modified with the nickel(II)bis(1,10-phenanthroline) complex and single-walled carbon nanotubes. Microchim Acta 183:1401-1408

11. Wu F, Huang T, Hu Y, Yang X, Ouyang Y, Xie Q (2016) Differential pulse voltammetric simultaneous determination of ascorbic acid, dopamine and uric acid on a glassy carbon electrode modified with electroreduced graphene oxide and imidazolium groups. Microchim Acta 183:2539-2546

12. Xing L, Ma Z (2016) A glassy carbon electrode modified with a nanocomposite consisting of $\mathrm{MoS}_{2}$ and reduced graphene oxide for electrochemical simultaneous determination of ascorbic acid, dopamine, and uric acid. Microchim Acta 183:257-263

13. Rao D, Zhang X, Sheng Q, Zheng J (2016) Highly improved sensing of dopamine by using glassy carbon electrode modified with $\mathrm{MnO}_{2}$, graphene oxide, carbon nanotubes and gold nanoparticles. Microchim Acta 183:2597-2604

14. Mei LP, Feng JJ, Wu L, Chen JR, Shen L, Xie Y, Wang AJ (2016) A glassy carbon electrode modified with porous $\mathrm{Cu}_{2} \mathrm{O}$ nanospheres on reduced graphene oxide support for simultaneous sensing of uric acid and dopamine with high selectivity over ascorbic acid. Microchim Acta 183:2039-2046

15. Ming L, Peng T, Tu Y (2016) Multiple enhancement of luminol electrochemiluminescence using electrodes functionalized with titania nanotubes and platinum black: ultrasensitive determination of hydrogen peroxide, resveratrol, and dopamine. Microchim Acta 183:305-310

16. Wang Y, Zhang Y, Hou C, Liu M (2016) Ultrasensitive electrochemical sensing of dopamine using reduced graphene oxide sheets decorated with $\mathrm{p}$-toluenesulfonate-doped polypyrrole/ $/ \mathrm{Fe}_{3} \mathrm{O}_{4}$ nanospheres. Microchim Acta 183:1145-1152

17. Xu MS, Liang T, Shi MM, Chen HZ (2013) Graphene-like twodimensional materials. Chem Rev 113:3766-3798

18. Yin PT, Shah S, Chhowalla M, Lee KB (2015) Design, synthesis, and characterization of graphene-nanoparticle hybrid materials for bioapplications. Chem Rev 115:2483-2531

19. Liu MM, Zhang RZ, Chen W (2014) Graphene-supported nanoelectrocatalysts for fuel cells: synthesis, properties, and applications. Chem Rev 114:5117-5160
20. Chen D, Feng HB, Li JH (2012) Graphene oxide: preparation, functionalization, and electrochemical applications. Chem Rev 112:6027-6053

21. Gao N, Fang XS (2015) Synthesis and development of graphene inorganic semiconductor nanocomposites. Chem Rev 115:8294 8343

22. Wang CI, Periasamy AP, Chang HT (2010) Photoluminescent Cdots@RGO probe for sensitive and selective detection of acetylcholine. Anal Chem 85:3263-3270

23. Shan CS, Yang HF, Han DX, Zhang QX, Ivaska A, Niu L (2010) Electrochemical determination of NADH and ethanol based on ionic liquid-functionalized graphene. Biosen Bioelectron 25:1504 1508

24. Wang Z, Xia J, Song D, Zhang F, Yang M, Gui R, Xia L, Bi S, Xia Y (2016) Lable-free quadruple signal amplification strategy for sensitive electrochemical p 53 gene biosensing. Biosen Bioelectron 77:157-163

25. Xia J, Cao X, Wang Z, Yang M, Zhang F, Lu B, Li F, Xia L, Li Y, Xia Y (2016) Molecularly imprinted electrochemical biosensor based on chitosan/ionic liquid-graphene composites modified electrode for determination of bovine serum albumin. Sensors Actuators B Chem 225:305-311

26. Wang Z, Li F, Xia J, Xia L, Zhang F, Bi S, Shi G, Xia Y, Liu J, Li Y, Xia L (2014) An ionic liquid-modified graphene based molecular imprinting electrochemical sensor for sensitive detection of bovine hemoglobin. Biosen Bioelectron 61:157-163

27. Xia J, Wang Z, Cai F, Zhang F, Yang M, Xiang W, Bi S, Gui R (2015) An electrochemical sensor for the sensitive detection of rutin based on a novel composite of activated silica gel and graphene. RSC Adv 5:39131-371317

28. Zhang HC, Huang H, Ming H, Li HT, Zhang LL, Liu Y, Kang ZH (2012) Carbon quantum dots $/ \mathrm{Ag}_{3} \mathrm{PO}_{4}$ complex photocatalysts with enhanced photocatalytic activity and stability under visible light. J Mater Chem 22:10501-10506

29. Hua S, Huang Q, Lin Y, Wei C, Ha Z, Zhang W, Guo Z, Bao X, Shi J, Hao A (2014) Reduced graphene oxide-carbon dots composite as an enhancedmaterial for electrochemical determination of dopamine dopamine. Electrochim Acta 130:805-809

30. Arcudi F, Đorđević L, Prato M (2016) Synthesis, separation, and characterization of small and highly fluorescent nitrogen-doped carbon NanoDots. Angew Chem Int Ed 55:2107-2112

31. Hu S, Huang Q, Lin Y, Wei C, Zhang H, Zhang W, Guo Z, Bao X, Shi J, Hao A (2014) Reduced graphene oxide-carbon dots composite as an enhanced material for electrochemical determination of dopamine. Electrochim Acta 130:805-809

32. Huang Q, Zhang H, Hu S, Li F, Weng W, Chen J, Wang Q, He Y, Zhang W, Bao X (2014) A sensitive and reliable dopamine biosensor was developed based onthe $\mathrm{Au} @$ carbon dots-chitosan composite film. Biosen Bioelectron 52:277-280

33. Laviron E (1979) General expression of the linear potential sweep voltammogram in the case of diffusionless electrochemical systems. J Electroanal Chem 101:19-28

34. Wang Y, Li Y, Tang L, Lu J, Li J (2009) Application of graphenemodified electrode for selective detection of dopamine. Electrochem Commun 11:889-892

35. Li SJ, He JZ, Zhang MJ, Zhang RX, Lv XL, Li SH, Pang H (2013) Electrochemical detection of dopamine using water-soluble sulfonated graphene. Electrochim Acta 102:58-65 